\title{
Elaborating the performance of Sensor Networks with merest energy to prolong network endurance
}

\author{
${ }^{1}$ M. Ramananthini, ${ }^{2}$ Mrs. Mary Joseph \\ ${ }^{1}$ PG Student, ${ }^{2}$ Assistant Professor, Anand Institute of Higher Technology, Kazhipattur, Chennai
}

\begin{abstract}
The routing process in Wireless Sensor Networks may differ from other normal routing methods because it contains no link to follow and no definite structure. The Wireless Sensor Networks is created by densely deployed sensor nodes. These nodes are powered by batteries.The energy-efficient coverage(EEC) problem is solved in other optimization algorithm but the idea of extending the lifetime capacity of the sensor networks fails when it communicates with several networks. The Bee Hive Optimization approach is used for increasing the lifetime of Wireless Sensor Networks. This generates a new path by taking energy as the fitness value to analyze different paths and provides best optimized path with low energy

Keywords: Bee-hive, network optimization, WSN.
\end{abstract}

\section{Introduction}

A Wireless Sensor Network consists of spatially distributed autonomous sensors to monitor physical or environmental conditions such as temperature, sound, pressure, etc. and to cooperatively pass their data through the network to a main location. The wireless sensor network is built of nodes from a few to several hundreds or even thousands where each node is connected to one sensor. Wireless Sensor Networks are used for a widevariety of applications. Wireless sensor networks are generally classified into structured and unstructured wireless sensor networks. In unstructured wireless sensor networks, humans cannot access directly. Most of the unstructured wireless sensor networks use batteries as an energy source. The important issuein unstructured wireless sensor networks is the energy-efficient coverage problem. It is a challenge for the traditional centralized, hierarchical or gridarchitecture to handle the large-scale and active resources.

A solution to Energy-efficient coverage problem is used to improve on the weakness of the conventional algorithm and it depends upon the characteristics of the sensors. Several optimization techniques have been proposed to increase the life of Wireless Sensor Networks. The Bee Hive optimization algorithm simulates the foraging behavior of bee colony for unconstrained optimization problems. The framework provides for fast convergence and includes the ability to determine random explorations.

A model of the bee hive clearly separates the self-organizing and decision-making behavior of the packets in the network and the problem-specific behavior of the packets outside the network is presented. The aim is to develop a simple model of the node applicable to solving problems in different domains. It includes Number of individuals in the network,Maximal time the packets can stay on the network, Maximal time the packets can stay in the routing table, probability that the packets observes wrong information about propagated source and consequently visits the source close to the propagated one. This approach is used to increase the lifetime of Wireless Sensor Networks and provides a better solution to overcome the weakness on energy efficiency coverage problem. It produces diverse search of solution and a better optimal solution with low computation complexity. In most applications a large number of sensor nodes are closely deployed and therefore communication between a sink and a node can be though intermediate nodes which are closer to each other. Such multihop communication uses short distance transmission and reduces energy consumption.

\section{Related Works}

In wireless communications design and characterizing energy efficiency is a demand which reduce the network construction and operating cost. [8] The concept of green architecture design is used and it needs a holistic consideration. It includes the view of deployment strategies for heterogeneous wireless networks. The green architecture design is analytically characterizing the open relations between energy efficiency and deployment efficiency. The development in networks and mobile terminals meets data traffic. The analysis of the energy efficiency of the best-select relaying is presented. [4] The single best relay is selected to relay the source message. The energy efficiency of a network is analyzed with distributed relay selection. Previously constrained circuit power consumption is used in the analysis of energy efficiency.

The concepts of optimal constellation size and transmission bandwidth are given. Duty-cycling provides additional delays in the message delivery process. The concept of adaptive staggered sleep protocol for efficient power management is targeted. The network topology is not required. The power management is determined. The wakeup scheduling is classified as on-demand, asynchronous and scheduled rendezvous schemes. [3] The 
adaptive staggered sleep protocol adjusts the activity of sensor nodes. Each sensor is capable to operate in active or standby modes which include different energy consumption rates. Greedy sensor deployment heuristics is considered. A mixed-integer linear programming formulation is used to minimize the use of energy in a certain period of time. The Lagrangian relaxation-based solution procedure is used. Binary integer programming formulation is determined to extend all the models. [10] The optimal sensor location is put together to maximize network lifetime. The task of MAC protocols is to avoid collisions from interfering nodes. The sensor MAC protocol is designed for wireless sensor networks. The performance of the sensor MAC is measured with latency and tradeoff. It provides long message into small fragments. The in-network data processing is an important feature of wireless sensor networks.

The evolutionary algorithms are used for solving complex problems. [1] The quantum inspired evolutionary algorithm is used to improve the performance and are solved for numerical problems. An optimization method is introduced. Cultural algorithms and co-evolutionary algorithms are included to evaluate many solutions. Multiple random access networks are used to operate on a different carrier frequency. Energy-efficient best selection relaying is used significantly in direct communications. It is used in realistic model. Optimal relay is used to maximize the efficiency. Adaptive modulation scheme is also used to determine efficiency. [3] The sensors are organized in the network and a novel genetic algorithm is introduced to sink each set of sensors. A novel genetic algorithm is adopted to allocate sinks. Homogeneous and heterogeneous networks are used. It minimizes the number of sinks. [5] In order to change the positions with time energy efficient sensor manager is considered. Multi objective optimization algorithm is used to provide better trade-off. Optimizations are obtained using mathematical programming and evolutionary algorithms. [6] The concept uses a recent variant of a powerful Multi-objective evolutionary algorithm known as Decomposition based Multi-objective evolutionary algorithm.

\section{Current and potential applications}

Current and potential applications of sensor networks include: military sensing, physical security, air Traffic control, traffic surveillance, video surveillance, industrial and manufacturing automation, distributed robotics, environment monitoring and building and structures monitoring. The sensors in these applications may be small or large, and the networks may be wired or wireless. Since, mobile wireless sensor networks are a relatively new concept; its specific, unique application areas are yet to be clearly defined. Most of its application scenarios are the same as that of traditional wireless sensor networks, with the only difference of mobility of mobile sink, preferably in the form of mobile phones.

\section{BHO Algorithm}

Bee Hive Optimization is an algorithm which is used to find the optimized path and thereby improve the efficiency of the network. It uses a preset number of packets to find the best of the sources by evaluating them and uses social interaction to agree upon the best source. This provides the clear separation of the decisionmaking mechanism inside the network from the problem-specific behavior of the packets outside the hive. The Bee Hive algorithm simulates the behavior of bee colony. When a data transferred from a node gets overloaded, it searches for the nearest node. From that node it transfers data with minimum energy.

The process consists of the following steps

Step: 1 Initialize the network in random fashion.

Step: 2 Calculate the fitness value for the network.

Step:3 Select the data for the neighborhood search.

Step:4 Find the fitness value and check for verification. If not satisfied the data will be abandoned and new packets are found out.

Step:5 Select the other packets.

Step: 6 Keep the data received by the nodes.

Conditions:

1 for $i=1, \ldots ., n s$

i scout[i]=Initialize $\operatorname{scout}()$

ii fp[i]=Initialize_fp(scout[i])

2 do until stopping condition=TRUE

i Recruit()

Different problems can be formulated considering the following design choices:

Sensor Deployment Method: deterministic versus random. A deterministic sensor placement may be feasible in friendly and accessible environments. Random sensor distribution is generally considered in remote or inhospitable areas, or for military applications. 
Sensing and Communication Ranges: WASN scenarios consider sensor nodes with same or different sensing ranges. Another factor that relates to connectivity is communication range, that can be equal or not equal to the sensing range.

Additional Critical Requirements: Energy-efficiency and connectivity. We will refer to these as energy-efficient coverage and connected coverage.

Algorithm Characteristics: Centralized versus distributed/localized.

Objective of the Problem: Coverage, maximum network lifetime or minimum number of sensors.

The context of the system refers to the various connections and relationships between the system and the environment. The proposed architecture exhibits energy coverage in wireless sensor networks in an unstructured environment. It also organizes itself to provide best possible outcome. The user requirement information is given in integer format. The user initializing the number of individuals. The fitness value is calculated and the best optimal path is identified. The new environment with new individuals is created.

The administrative requirements help in defining and framing the functions of the sensors. All the decisions are made by the central unit. It uses multi-agent system where individuals communicate to make own decisions. It has to take the source and also to return the source. The sensor is meant to cover a regular distance between the sensor and the point of interest within the sensing range of sensors.

These requirements determine the resources required, response time, network lifetime, computation time, energy efficiency and performance of the system.

The Bee-Hive Optimization Approach is simulated mainly on NS2 simulator. Network Simulator (Version 2), widely known as NS2, is simply an event driven simulation tool that has proved useful in studying the dynamic nature of communication networks. Simulation of wired as well as wireless network functions and protocols (e.g., routing algorithms, TCP, UDP) can be done using NS2. In general, NS2 provides users with a way of specifying such network protocols and simulating their corresponding behaviors. The main obstacle in the coordination with other networks is limited energy of sensor nodes. To monitor the Wireless Sensor Networks, the data produced by the sensor nodes should be accessible...

\section{Implementation}

\subsection{Network Construction}

The number of nodes are created and connected with respect to some protocols. That node is considered as a sensor node. The characteristics are predefined like node size, packet size etc., the packet size depends on the number of nodes in network setup. The routing of packet transmission between the nodes is created with the help of TCL scripting.

\subsection{Neighborhood Search}

Each node keeps the count of number of messages of its neighbors. Each node has to announce the number of packets it has forwarded over the particular time. The neighborhood node collects the number of forwarded packets by the sender and the comparator node. It compares the result announced by the sender and the neighbor. Nearest neighbor node is considered as a guard node which is located at the shortest path.

\subsection{EEC problem identification}

When multiple nodes communicate, the unwanted nodes are removed. The network checks for communication link twice for range. This checking takes place at the exact position. The process occurs within a particular time and at a particular distance with the help of scheduling.

\subsection{Structured node formation}

New nodes are created. Initially the nodes are rotated randomly. Once data is transferred at a particular path with minimum energy then the selected path is fixed for further transmission. Again if another node wants to transmit data it uses the fixed path and transfers data along that path. After all nodes are formed using the optimization algorithm the lifetime is increased. The unwanted nodes are considered as a dummy node instead of being removed. Hence it saves time and energy.

\section{The simulation results can be summarized as follows:}

For a specific number of targets, the network lifetime output by our heuristics increases with the number ofsensors and the communication range and for a specific number of sensors and sensing range, the network lifetime decreases as the number of targets to be monitored increases.

The most important challenge in designing algorithms is mobility and limited battery capacity of nodes.Mobility of nodes results in continuously evolving new topologies and consequently the routing algorithms have to discover or update the routes in real time but with small control overhead. The limited battery capacity requires that the packets if possible be distributed on multiple paths, which would result in the 
depletion of batteries of different nodes at an equal rate and hence as a result the life time of networks would increase. Thus to design a routing algorithm that is not only energy-efficient but also delivers performance is an important challenge in routing protocols. To reduce the computation time, however, is less important than to prolong the network lifetime in solving the EEC problem. The reason is that the EEC problem is not the optimization problem demanding the solution in real time. Many algorithms have been proposed to solve the EEC problem. The EEC problem was converted into a binary integer programming problem. Simulation results are conducted to verify the effectiveness of the algorithm for solving the EEC problem in comparison with existing algorithms. Sink node is source. It is similar to head node which gather, controlled data collected by the other sensor node. The WSN consists of sink node referred to as base station. Node to node delivery is a general networking reference and is often contrasted with end to end delivery. A network is uniformly made of connection between many points. The Probability sensor detection model represents a more realistic approach in solving the problem.

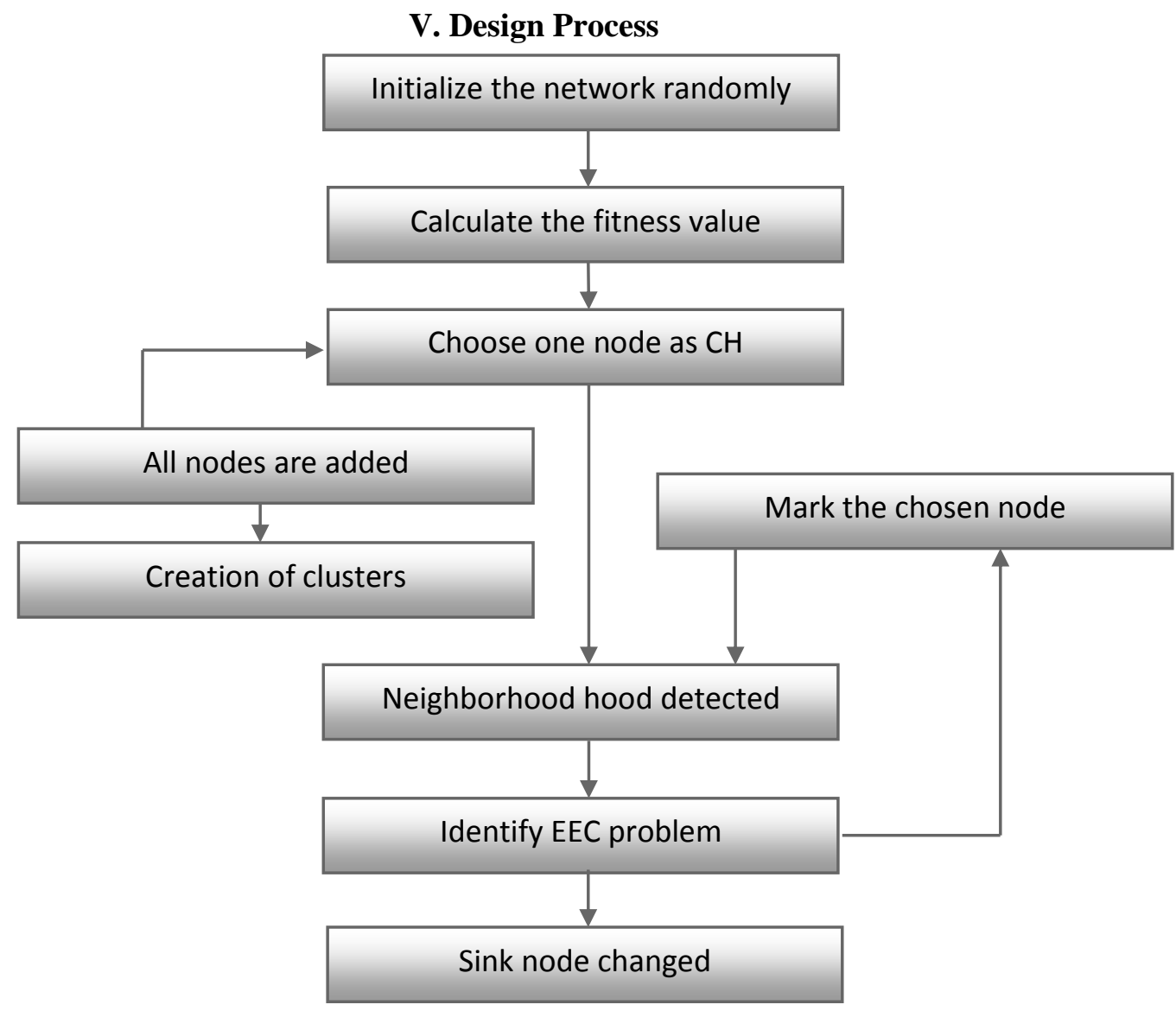

Fig. 1. Process diagram of the proposed algorithm

The properties of the algorithm includes

Autonomous: the capability of performing autonomous actions.

Proactive: the capability of exhibiting opportunistic and goal-oriented behaviorand taking initiative where appropriate.

Responsiveness: the capability of perceiving the environment and responding ina timely fashion to the changes that occur in it.

Round and waggle dances serves the recruitment and reactivation of foragers. Interestingly it is distinguishes between two distance ranges. Every node is a beehive, with packets (bees) collecting data (food). This means that every packet is a very simple mobile agent, transporting user data and route information from its starting node to its destination. Transmitting packets is obviously very costly. Overhead of the routing algorithm and retransmissions due to a wrong or broken route has to be minimized. Another factor is the operating mode of the network interface. Promiscuous mode requires a lot of energy (overhearing) but may gather a lot of useful information about the surrounding nodes. 


\section{Conclusion}

With minimum energy, maximum work is done. This algorithm provides better coverage than Jengainspired algorithm. This paper presents a Bee Hive Optimization algorithm to increase the lifetime of wireless sensor network performs better than other algorithms like ACO, PSO and Jenga-Inspired Optimization Algorithm.

\section{Future Works}

The future work targets at implementing security to the network and prevents attacks. The future developments in sensor networks would produce very powerful and cost effective devices, so that they may be used inapplications like underwater acoustic sensor systems, sensing based cyber physical systems, time critical applications, cognitive sensing and spectrum management, and security and privacy management. One can envision a future in which wireless devices, such as wireless keyboards, power point presenters, cell phone headsets and health monitoring sensors will be ubiquitous.

\section{References}

[1] Andre Vargas Abs da Cruz, Marley M. B. R. Vellasco, Marco Aurelio C. Pacheco, (2010)"Quantum-Inspired evolutionary algorithms applied to numerical optimization problems", Applied Computational Intelligence, Pontifical Catholic University of Rio de janeiro, Brazil.

[2] Department of Computer Science, Sun yat-Sen University, Guangzhou, China. Gibing Lim, Leonard J.cimini, Jr., (2012) "Energyefficient co-operative relaying in heterogeneous radio access networks", IEEE Wireless communication letters, VOL. 1, NO. 5.

[3] Giuseppe Anastasi, Marco Conti, and Mario Di Francesco, (2009) "Extending the lifetime of wireless sensor networks through adaptive sleep", IEEE transactions on industrial informatics, Vol. 5, NO. 3.

[4] Gubong Lim and Leonard J. Cimini, Jr, (2008) "Energy-efficient best-select relaying in wireless cooperative networks", University of Delaware, Newark.Huawei Technologies, Co. Ltd., Shanghai, China, IEEE.

[5] Jiming Chen, Junkun Li, Shibo He, Youxian Sun, Hsiao-Ha Chen, (2010) "Energy-efficient coverage based on probabilistic sensing model in wireless sensor networks", IEEE Communications letters, VOL. 14, NO. 9.

[6] Soumyadipsengupta, Swagatam Das, Md. Nasir, Athanasiosv. Vasilakos and Witold Pedryez, (2012) "Energy-efficient differentiated coverage of dynamic objects using an improved evolutionary multi-objective optimization algorithm with fuzzy dominance", WCCI 2012 IEEE World Congress on computational intelligence.

[7] Wei Ye, John Heinemann, Deborah String, (2004) "Medium access control with coordinated adaptive sleeping for wireless sensor networks", IEEE/ACM Transactions on networking, VOL. 12, NO. 3.

[8] Yan Chen, Shunqing Zhang and Shugong Xu, (2010) "Characterizing Energy Efficiency and Deployment Efficiency Relations for Green Architecture Design", Huawei Technologies, Co. Lt., IEEE.

[9] YavuzBogacTurkogullari, Necati Aras, I. Kuban Altinel, Cem Ersoy, (2007) "Optimal placement and activity scheduling to maximize coverage lifetime in wireless sensor networks", Bogazici University, Bebek, Istanbul, Turkey, IEEE

[10] Ying Lin, Xiao-min Hub, Jun Zhang, Ou Liu, Hai-Lin Liu, (2007) "Optimal node scheduling for the lifetime maximization of two-tier Wireless sensor networks”, Development of Computer Science, Sun Yat-Sen University. 